\title{
A Next-Generation Friction Burn Caused by a Cordless Vacuum Cleaner
}

\author{
Sung Jae Wee (D), Myong Chul Park (D) \\ Department of Plastic and Reconstructive Surgery, Hallym University Sacred Heart Hospital, Hallym University College of Medicine, Anyang, Korea
}

\begin{abstract}
Consumers today are increasingly using a new type of vacuum cleaner with a soft roller head. Among injuries from the use of household appliances, vacuum cleaner-related friction burns tend to be underestimated in their severity, compared to treadmill-related friction burns, which have been explored more extensively in literature. We present the case of a 17-month-old child with a whitish eschar on the volar side of her right fourth toe, which had been sucked into the rotating soft roller head of one of the new cordless vacuum cleaners. Initially, the wound resembled a simple abrasion, but it was whitish enough for us to suspect a full-thickness burn. We performed gauze rubbing for granulation with a biologic dressing material and basic fibroblast growth factor. The burn took more than 3 weeks to heal completely. It is essential to reappraise the severity of friction burns caused by the more recent vacuum cleaner models with powered rollers and to establish appropriate treatment protocols.
\end{abstract}

Keywords: Burns; Friction; Vacuum

\section{Introduction}

Friction burns are commonly encountered in motorcycle or bicycle riders in traffic accidents. Other common causes include sports injuries, the use of an exercise treadmill at home, fast-moving belts in factories, tourniquets applied over the thigh without proper padding, and domestic vacuum cleaners, the last causing friction burns in young children [1].

As more people exercise at home with fitness equipment, there have been more reports of treadmill-related burn injuries in children [2]. Most are deep partial-thickness or full-thickness burns, requiring a surgical approach such as debridement or skin grafting [3]. As a result of the abundance of reports on treadmill-related friction burns, appropriate treatment protocols are well established, whereas fewer cases have been reported on friction burns caused by vacuum cleaners, considered to be of less importance or severity [3].

We describe a pediatric case of a full-thickness friction burn caused by a powerful vacuum cleaner with a soft roller head. The newly developed vacuum cleaners pose a risk of severe burns that warrant more attention from clinicians as a new generation of friction burns. The study was approved by the Institutional Review Board of Hallym Sacred Heart Hospital (IRB No. 2019-09-018) and performed in accordance with the principles of the Declaration of Helsinki. Written informed consent was obtained from the patient's guardians.

\section{Case Report}

Received: June 26, 2020

Revised: August 2, 2020

Accepted: August 3, 2020

Corresponding author: Myong Chul Park, M.D., Ph.D.

Department of Plastic and Reconstructive Surgery, Hallym University Sacred Heart Hospital, Hallym University College of

Medicine, 22 Gwanpyeong-ro 170beon-gil,

Dongan-gu, Anyang 14068, Korea

Tel: +82-31-380-3781

Fax: +82-31-380-5980

E-mail: mpark@hallym.or.kr

This is an Open Access article distributed under the terms of the Creative Commons Attribution Non-Commercial License (https://creativecommons.org/licenses/by-nc/4.0/) which permits unrestricted non-commercial use, distribution, and reproduction in any medium, provided the original work is properly cited.

(c) 2020 Korean Wound Management Society 


\section{Case}

A 17-month-old child visited the outpatient department with a whitish eschar on the volar side of her right fourth toe (Fig. 1). The eschar measured approximately $1.0 \times 1.0 \mathrm{~cm}$, and the wound had been caused by the toe being sucked into the rotating soft roller head of a cordless vacuum cleaner 7 days previously. Though the wound had been dressed by a local pediatrician, the patient was referred to our hospital because of poor healing. The wound had poor circulation and was whitish enough for us to suspect a deep second- or third-degree burn.

We initially dressed the wound with gauze rubbing and applied basic fibroblast growth factor (Fiblast Spray; Kaken

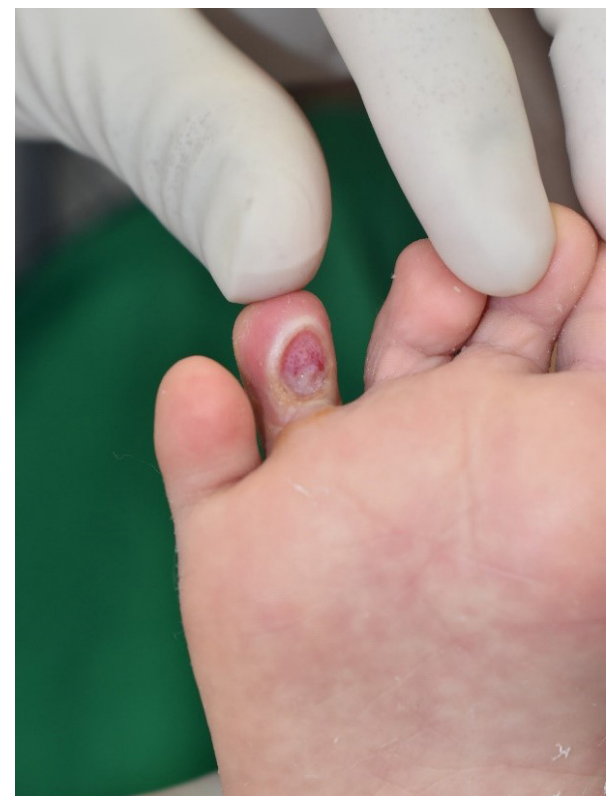

Fig. 1. A friction burn wound at postburn day 14 .

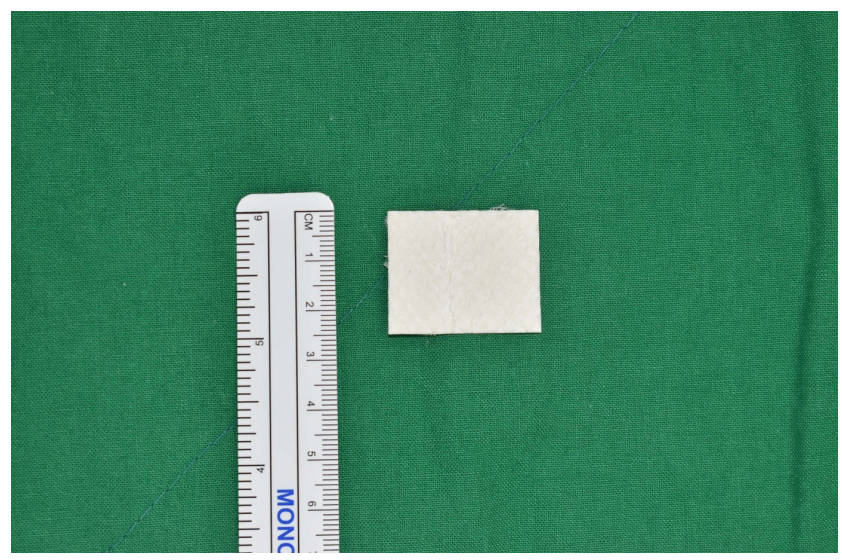

Fig. 2. A biologic dressing material, fish skin.
Pharmaceutical, Tokyo, Japan) treatment for better granulation over the course of 1 week [4].

As the wound had not healed sufficiently by 2 weeks after the injury, we decided to use fish skin as a biologic dressing material (Kerecis Omega-3 Wound; Alvogen, Seoul, Korea) (Fig. 2). The combination of the biologic material and topical spray dressing led to increased granulation, resulting in re-epithelialization, after which a hydrocolloid dressing was placed (Fig. 3).

A follow-up examination performed 2 months later showed no lingering wound or complications (Fig. 4).
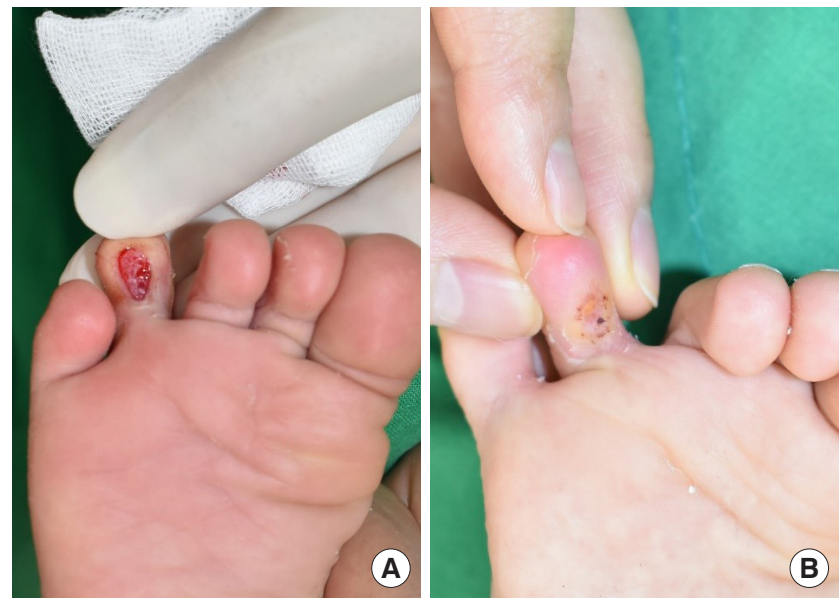

Fig. 3. Gross photographs. (A) The wound at postburn day 16 after gauze rubbing and biologic material dressing. (B) The wound at postburn day 26 showing complete healing.

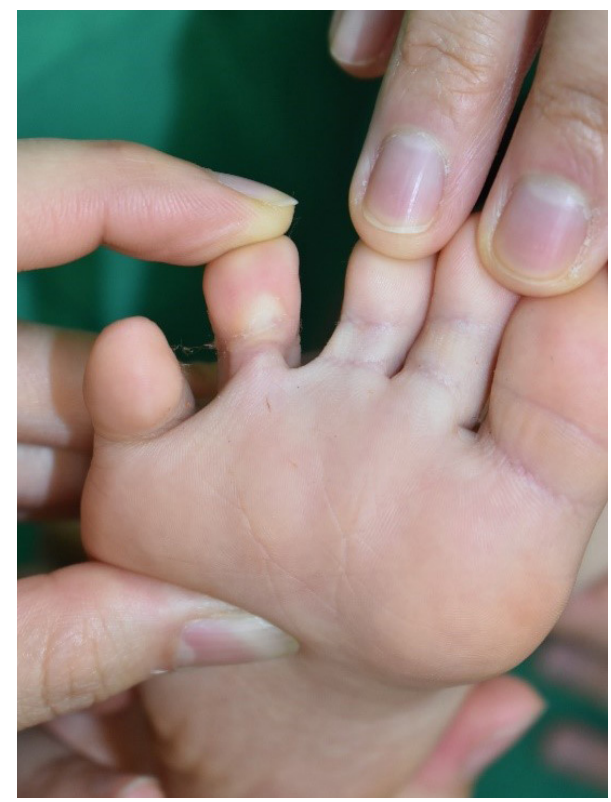

Fig. 4. The wound at postburn day 61 . 


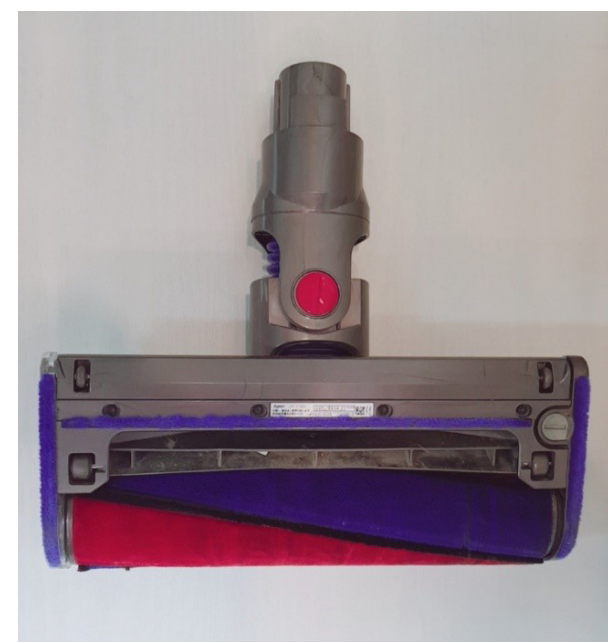

Fig. 5. A soft roller head of cordless vacuum cleaner.

\section{Discussion}

Friction burns in children due to commonplace domestic appliances are a cause of major concern as they are steadily increasing in numbers. Children's hands and toes are vulnerable to friction burns because their skin is thinner than that of adults and often lacks callus formation [5]. Friction burns caused by home exercise equipment, such as treadmills, have been widely reported for decades. In contrast, research on vacuum cleaner-related friction burns has been insufficient [3], and the severity of these wounds may therefore be underestimated on initial evaluation.

In particular, a recently developed type of digital motorpowered vacuum cleaner with a soft roller head is increasingly replacing traditional vacuum cleaners, which emphasized the power of suction itself. It was in 2004 that Dyson introduced the new vacuum cleaner equipped with a "powered roller" head (Fig. 5) to provide better functionality than the traditional cleaners. This roller was designed because simply improving the suction power of the vacuum cleaner, which had been previously considered integral to the cleaner's performance, did not actually improve its ability to suction dust. The company introduced the powered roller as a mechanism to collect dust and sweep it to the front of the vacuum cleaner head's inlet for easier suction, enhancing performance and making cleaning easier. As most vacuum cleaner companies have launched similar models, children are now more likely to accidentally touch these soft roller heads with their fingers or toes when the vacuum cleaner is on and suctioning. The rotating powered roller can then cause deeper friction burns than those by previous generations of vacuum cleaners. The vacuum cleaner that caused the injury in the present case has a digital motor that rotates at up to 125,000 revolutions per minute, and the suction force of the soft roller head reaches 185 airwatts, a measure of airflow and the amount of power that a vacuum cleaner produces and uses. Though the initial wound looked like a simple scratch or abrasion, and the child's grandfather stated that the duration of contact between the child's toe and the running vacuum cleaner was very brief, still the burn required more than 3 weeks of treatment. This shows the strength of the friction-induced by the roller head and the suction force on the child's toe.

Only a few years ago, vacuum cleaner-related friction burns were thought to be less severe than those caused by the firm rubberized belt of a treadmill, as the motors that rotated the nylon bristles on a vacuum cleaner's brush cylinder were less powerful than those used in treadmills [3,6,7]. However, conservative treatment may not be sufficient for burns caused by strong friction with the antistatic fine carbon-fiber filament on the soft roller heads in the more recent vacuum cleaner models. These wounds may require a surgical approach or biologic material dressing. It is therefore essential to reappraise the severity of friction burns caused by the newly developed vacuum cleaners.

In cultures where uncarpeted hardwood floors are more common than carpet flooring, people are more likely to prefer this new type of vacuum cleaners with a powered roller, because it sweeps dust to the inlet of the vacuum cleaner's head, making cleaning easier and more effective. Friction burns caused by this equipment will be more frequently encountered in the future. Our case report may serve as a reference for the treatment protocol for a friction burn caused by a vacuum cleaner with a soft roller head. We also recommend that clinicians be aware of the risks of the next-generation injuries caused by domestic appliances with new and more powerful features.

\section{Conflict of interest}

No potential conflict of interest relevant to this article was reported.

\section{ORCID iDs}

Sung Jae Wee

Myong Chul Park

https://orcid.org/0000-0002-2397-7450

https://orcid.org/0000-0002-0845-2672 


\section{References}

1. Agrawal A, Raibagkar SC, Vora HJ. Friction burns: epidemiology and prevention. Ann Burns Fire Disasters 2008; 21:3-6.

2. Goltsman D, Li Z, Connolly S, et al. Pediatric treadmill burns: assessing the effectiveness of prevention strategies. Burns 2016;42:1581-7.

3. Marchalik R, Rada EM, Albino FP, et al. Upper extremity friction burns in the pediatric patient: a 10-year review. Plast Reconstr Surg Glob Open 2018;6:e2048.
4. Hayashida K, Fujioka M, Morooka S, et al. Effectiveness of basic fibroblast growth factor for pediatric hand burns. J Tissue Viability 2016;25:220-4.

5. Friedrich JB, Muzaffar AR, Hanel DP. Pediatric hand friction burns from treadmill contact. Hand (N Y) 2007;2:18893.

6. Macgregor D. Hand injuries in young children from contact with vacuum cleaners. Emerg Med J 2002;19:80-1.

7. Grob M, Josty IC, Soldin MG, et al. Paediatric friction hand injuries caused by domestic vacuum cleaners-a review from one unit. Burns 2003;29:714-6. 